\title{
Edad materna y malformaciones congénitas. Un registro de 35 años. 1970-2005
}

\author{
Julio Nazer $\mathbf{H}^{1}$, Lucía Cifuentes $\mathbf{O}^{2}$, Alfredo Águila $\mathbf{R}^{1}$, \\ Pilar U reta La, María Piedad Bello Pb, Francisca Correa $C^{b}$, \\ Francisco Melibosky $\mathbf{R}^{\mathrm{c}}$.
}

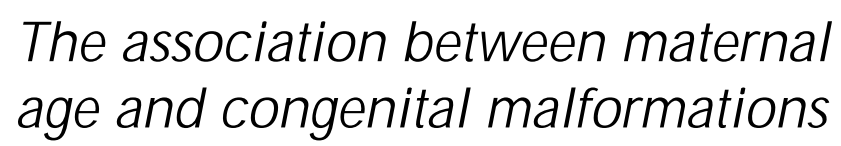

Background: There is an association between extreme maternal ages and the risk of congenital malformations. This is specially true for chromosomal abnormalities among women of advanced ages and disruptive malformation among teenage mothers. Aim: To determine the association between maternal ages and incidence of congenital malformations at the obstetric ward of a clinical hospital. To compare these figures with those of Chile. Material and methods: The hospital registries of the Latin American Collaborative Study of Congenital Malformations (ECLAMC) between 1996 and 2005, were consulted. This is a database of all marformations detected in newborns at the hospital. Results: An overall prevalence of malformations of $8,4 \%$ was detected at the hospital. There is a significantly lower frequency of mothers aged less than 20 years than in the rest of Chile. Mothers aged between 20 and 29 years have the lower frequency of malformed children. Women aged less than 20 years and over 39 years of age, account for $56 \%$ of malformed children. Maternal ages and the rates of malformations, increased in a parallel fashion at a rate of 0.2 years and 2.2 malformed children per 1,000 born alive, per calendar year, respectively. Conclusions: The association between prevalence rates of congenital malformations and maternal age is $U$ chaped with a higher proportion of malformed children among women aged less than 20 years or more than 39 years (Rev Méd Chile 2007; 135: 1463-69).

(Key words: Abnormalities; Congenital defects; Maternal age)

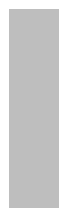

\footnotetext{
Recibido el 29 de septiembre, 2006. Aceptado el 27 de noviembre, 2006.

${ }^{1}$ Unidad de Neonatología, Hospital Clínico de la Universidad de Chile. ${ }^{2}$ Instituto de Ciencias Biomédicas, Facultad de Medicina. Universidad de Chile. Santiago de Chile.

aMédico Becario del Servicio de Genética, Hospital Clínico de la Universidad de Chile.

bInternas de Medicina, Universidad de Chile.

${ }^{\mathrm{c}}$ Interno de Medicina, Universidad Mayor.
}

Correspondencia a: Dr. Julio Nazer H. Unidad Neonatología, Hospital Clínico Universidad de Chile. Santiago de Chile. Fono: 7161668.

E mail: julionazer@mi-mail.cl 
$\mathrm{L}$ os promedios de las edades maternas en la maternidad del Hospital Clínico de la Universidad de Chile (HCUCH) han ido aumentando progresivamente desde 25 años en 1972, a 27,8 años en 1988, 29,6 años en 2002, para luego bajar a 27,7 en $2005^{1}$. Ello ha sido provocado por el aumento de nacimientos en los grupos de mujeres de más de 30 años, al mismo tiempo que han disminuido los de las menores de 20 años.

La asociación entre las edades maternas extremas y malformaciones congénitas (MC) ya ha sido comprobada por muchos autores ${ }^{2-7}$. Además se ha encontrado que las edades maternas avanzadas se relacionan principalmente con MC de origen cromosómico producidas por no disyunción ${ }^{10}$ y dentro de este grupo se destacan las trisomías, como las trisomías 13,18 y $21^{1,11-16}$. También se ha descrito un mayor riesgo de defectos del tubo neural, especialmente anencefalia y espina bífida en hijos de mujeres mayores de 40 años $^{16}$. Las edades maternas más jóvenes se han relacionado con MC no cromosómicas, ya sea de origen disruptivo, como gastrosquisis $^{7,8,11,13,15}$, como también con defectos de otro tipo, como estenosis pilórica 7,8,16,17, hidrocefalia, polidactilia, persistencia del ductus arterioso ${ }^{7,8,12}$, displasia septo-óptica ${ }^{13,18}$. Esta asociación implica que los hábitos de las mujeres jóvenes, como alimentación, consumo de tabaco y drogas, pudieran corresponder a factores de riesgo para estos defectos ${ }^{15}$. Croen y Shaw $^{8}$ encontraron en una revisión de más de un millón de nacimientos, que el riesgo para malformaciones de origen cromosómico de las mujeres de menos de 20 años era comparable con las de mayor edad. Encontraron también que varias MC, como gastrosquisis y estenosis pilórica presentaban frecuencias mayores que en el grupo de 25 a 29 años, lo que ya había sido comunicado anteriormente ${ }^{8,19}$.

Otros factores de importancia que tienen influencia en la morbimortalidad de los hijos de las mujeres de edades extremas son baja edad gestacional y bajo peso de nacimiento, hechos que se ven con mayor frecuencia en esos grupos etarios $^{6,8,18}$. Se ha visto también que todos estos factores pueden encontrarse en mujeres jóvenes, casadas, con buen nivel sociocultural, que reciben un adecuado control prenatal, sugiriendo que la edad materna baja aumenta intrínsecamente el riesgo de un resultado adverso en el embara$\mathrm{zO}^{19,20}$.

\section{OBjetivos}

Los objetivos de esta investigación fueron establecer la distribución de los nacimientos de la maternidad del HCUCH según las edades maternas y compararlas con las de la población general de Chile ${ }^{21}$.

Construir una curva de la evolución de los promedios de edad materna de la población atendida en la maternidad del HCUCH desde 1970 hasta 2005.

Construir una curva de la evolución de las tasas anuales de prevalencia al nacimiento de las malformaciones congénitas (MC) desde 1970 hasta 2005.

Superponer ambas curvas en un gráfico y estudiar la relación que hubiera entre ellas.

Establecer la frecuencia de las MC en cada grupo etario estudiado, calcular el riesgo de tener hijos con defectos congénitos en los distintos grupos etarios y tratar de individualizar a aquellas MC que se presenten con mayor frecuencia en los extremos de la curva.

\section{PACIENTES Y MÉTODO}

El HCUCH participa desde 1969 en el Estudio Colaborativo Latino Americano de Malformaciones Congénitas (ECLAMC) y registra a todos los recién nacidos $(\mathrm{RN})$, vivos y mortinatos de 500 gramos o más que presenten una o más MC. Son ingresados a una base de datos con una ficha electrónica ad-hoc en la que se consignan fecha de nacimiento, sexo, peso, edad de la madre, diagnóstico y descripción de la o las malformaciones, su estado al alta, además de otros antecedentes demográficos. Se toma como control al recién nacido vivo (RNV) del mismo sexo, sin defectos congénitos, que nace a continuación ${ }^{22}$.

Se estudiarán todos los RN malformados, vivos o mortinatos que nacieron desde el $1^{\text {o }}$ de enero de 1996 hasta el 31 de diciembre de 2005. Se distribuirán según la edad de sus madres, a su vez agrupadas de la siguiente forma:
$<15$ años
30-34 años
15-19 años
35-39 años
20-24 años
40-44 años
25-29 años
$45 \mathrm{y}+$ años 
Se comparará la distribución de las edades maternas en el HCUCH con la distribución en todo Chile mediante prueba de $\chi^{2}$.

Se calculará la prevalencia al nacimiento de MC para cada uno de los estos grupos de edades maternas y se calculará el riesgo relativo de tener un hijo malformado en cada uno de estos intervalos etarios.

Se estudiará el comportamiento de las tasas de malformaciones congénitas y el promedio de edad materna, año a año, en la maternidad del HCUCH. Se estimará la velocidad con que estos estimadores han crecido a lo largo del tiempo a través de una regresión lineal y se estudiará la correlación entre ambas variables.

Para identificar las MC específicas que pudieran contribuir a aumentar la prevalencia entre las mujeres jóvenes y de edad avanzada, las compararemos con el grupo etario entre 20 y 29 años de edad. Escogimos este grupo como referencia debido a que tienen la menor frecuencia de MC y el mayor número de nacimientos.

\section{Resultados}

En el período 1996-2005 ocurrieron 21.130 nacimientos consecutivos en la maternidad del Hospital Clínico de la Universidad de Chile (HCUCH) 20.972 nacidos vivos (NV) y 158 mortinatos (MM), lo que representa una mortinatalidad de $0,76 \%$. En el total de nacimientos se encontró 1.767 recién nacidos (RN) que eran portadores de una o más malformaciones congénitas (MC) (8,4\%), $1.733 \mathrm{NV}$ $(8,3 \%)$ y $34 \mathrm{MN}(21,5 \% ; 34 / 158)$.

La Tabla 1 muestra la distribución del total de nacimientos en Chile el año 2003 por grupos de edades maternas, según el Ministerio de Salud y el INE (Instituto Nacional de Estadísticas) y la distribución observada en el HCUCH entre los años 1996 y 2005; ambas distribuciones son significativamente diferentes, ya que en este último hay menor cantidad de madres menores de 20 años que en el resto de Chile. $\left(\chi^{2}=1312,1, p<0,00001\right)$.

En la Tabla 2 se observa la distribución del total de nacimientos y de los RN malformados, de la maternidad del $\mathrm{HCUCH}$, según los grupos de edades maternas. Las tasas de prevalencia al nacimiento de MC en los RN son significativamente distintas en todos los grupos de edades maternas $\left(\chi^{2}=0,00001\right)$. Las madres entre 20 y 29 años tienen con menor frecuencia hijos malformados que todas las otras madres. Se puede apreciar que las mujeres menores de 20 años y mayores de 39 años (9,7\% de las mujeres), producen el 55,8\% de los RN malformados. Es decir estos pequeños grupos de mujeres de edades extremas concentran más de la mitad de los niños con malformaciones congénitas (23,9\% las menores de 20 años y $31,9 \%$ las mayores de 39 años). El grupo etario que proporcionalmente presentó menor prevalencia al nacimiento de MC, fue el de 25 a 29 años (7,3\%), seguido por el de 20 a 24 años $(7,8 \%)$.

En la Tabla 3 se muestra la proporción de hijos malformados en las madres de cada grupo etario. Las tasas de prevalencia al nacimiento de malfor-

Tabla 1. Total de nacimientos distribuidos por grupos de edades maternas en Chile (2003) y en H ospital C línico de la Universidad de Chile (H U CH ) (1996-2005)

\begin{tabular}{|lrrrr|}
\hline & \multicolumn{2}{c}{$\begin{array}{c}\text { Chile. 2003* } \\
\text { Número de } \\
\text { Edad materna (años) }\end{array}$} & $\%$ & \multicolumn{2}{c|}{$\begin{array}{c}\text { Número de } \\
\text { recién nacidos }\end{array}$} & $\%$ \\
\hline$<$ de 15 & 994 & 0,42 & 34 & 0,16 \\
$15-19$ & 33.838 & 14,43 & 1.193 & 5,64 \\
$20-34$ & 161.536 & 68,88 & 16.008 & 75,76 \\
35 y + & 38.116 & 16,25 & 3.893 & 18,42 \\
Total: & 234.486 & 100,00 & 21.128 & 100,00 \\
\hline
\end{tabular}

* Ministerio de Salud 
Tabla 2. Total de nacimientos por grupos de edad materna, porcentaje de $R \mathbf{N}$ en cada intervalo etario, número y tasa de RN malformados por cien nacimientos. H ospital C línico Universidad de Chile. Período 1996-2005

\begin{tabular}{|lrrrr|}
\hline Edad materna & Nacimientos & $\%$ & RN malf. & Tasa \% \\
\hline$<$ de 15 años & 34 & 0,2 & 5 & 14,7 \\
$15-19$ & 1.193 & 5,6 & 110 & 9,2 \\
$20-24$ & 4.094 & 19,4 & 320 & 7,8 \\
$25-29$ & 6.432 & 30,5 & 468 & 7,3 \\
$30-34$ & 5.482 & 26.0 & 475 & 8,5 \\
$35-39$ & 3.059 & 14,5 & 286 & 9,3 \\
$40-44$ & 784 & 3,7 & 93 & 11,9 \\
45 y & 50 & 0,2 & 10 & 20,0 \\
Total & $21.128^{*}$ & & 1.767 & 8,4 \\
\hline
\end{tabular}

*En 2 casos no se consignó la edad materna.

Tabla 3. D istribución del total de nacimientos, de RN malformados, proporción, riesgo relativo de tener un hijo malformado, con sus respectivos intervalos de confianza en los distintos grupos de edades maternas. H ospital Clínico Universidad de Chile. Período 1996-2005

\begin{tabular}{|lrrrrr|}
\hline Edad materna & RN totales & RN malf. & proporción & RR & $\begin{array}{r}\text { Intervalo de } \\
\text { Conf. } 95 \%\end{array}$ \\
\hline$<$ de 15 años & 34 & 5 & $1 / 6,8$ & 1,7 & $0,7-4,3$ \\
$15-19$ & 1193 & 110 & $1 / 10,8$ & 1,3 & $1,1-1,6$ \\
$20-24$ & 4094 & 320 & $1 / 12,8$ & 1,1 & $1,0-1,3$ \\
$25-29$ & 6432 & 468 & $1 / 13,7$ & 1 & \\
$30-34$ & 5482 & 475 & $1 / 11,5$ & 1,2 & $1,1-1,4$ \\
$35-39$ & 3059 & 269 & $1 / 11,4$ & 1,3 & $1,1-1,5$ \\
$40-44$ & 784 & 93 & $1 / 8,4$ & 1,6 & $1,3-2,0$ \\
$45 \mathrm{y}+$ & 50 & 10 & $1 / 5$ & 2,0 & $1,0-4,0$ \\
Total & $21.128^{*}$ & 1.767 & $1 / 12$ & & \\
\hline
\end{tabular}

* En 2 casos no se consignó la edad materna.

maciones en los RN son significativamente distintas en todos los grupos de edades maternas $\left(\chi^{2}=\right.$ $30,3, \mathrm{p}<0,00001)$. Se han estimado también los riesgos relativos para cada intervalo de edad materna, tomando como referencia las madres entre 25 y 29 años, que son las que tienen el mayor número de nacimientos y que presentan con menor frecuencia un hijo malformado. Se observa que el riesgo relativo es mayor en las edades extremas, especialmente en las mujeres de más de 39 años.
Al agrupar las MC por sistemas, se encontró que las malformaciones del sistema gastrointestinal ( $\mathrm{p}=0,02)$, malformaciones múltiples y síndrome de Down $(\mathrm{p}=0,03)$ eran más frecuentes entre las madres de edades mayores, en tanto que entre los hijos de madres adolescentes son más frecuentes las malformaciones craneofaciales y gastrosquisis ( $\mathrm{p}=0,02$; 0R: 1,5; IC al 95\%: 1,04-2,17). El $13,8 \%$ de los RN malformados eran PEG, 46,6\% AEG y $8,3 \%$ GEG. 
Hubo 2 casos en que no se consignó la edad materna.

La Figura 1 muestra con claridad que las edades extremas de la curva presentan las tasas mayores de prevalencia al nacimiento de MC. En efecto, es la típica curva en "U", en que las edades sobre 39 años y las menores de 20 concentran la mayor proporción de RN con defectos congénitos.

La Figura 2 muestra la evolución de las tasas de prevalencia de malformaciones congénitas y de los promedios de edad materna, anualmente, desde
1969 a 2005. Se aprecia que ambas variables aumentan paulatinamente a lo largo del tiempo; al construir la recta de regresión mínimo cuadrática de cada una de las curvas se encuentra que ambas tienen similar pendiente positiva ( $p>0,05)$ : 0,16 años de incremento de edad materna promedio por cada año calendario $(\mathrm{p}<0,0001)$ y un incremento anual de 2,21 recién nacidos malformados por 1.000, en la tasa de prevalencia $(p<0,0001)$. El coeficiente de correlación de Pearson entre ambas variables es 0,857 , altamente significativo ( $\mathrm{p}<0,001$ ).

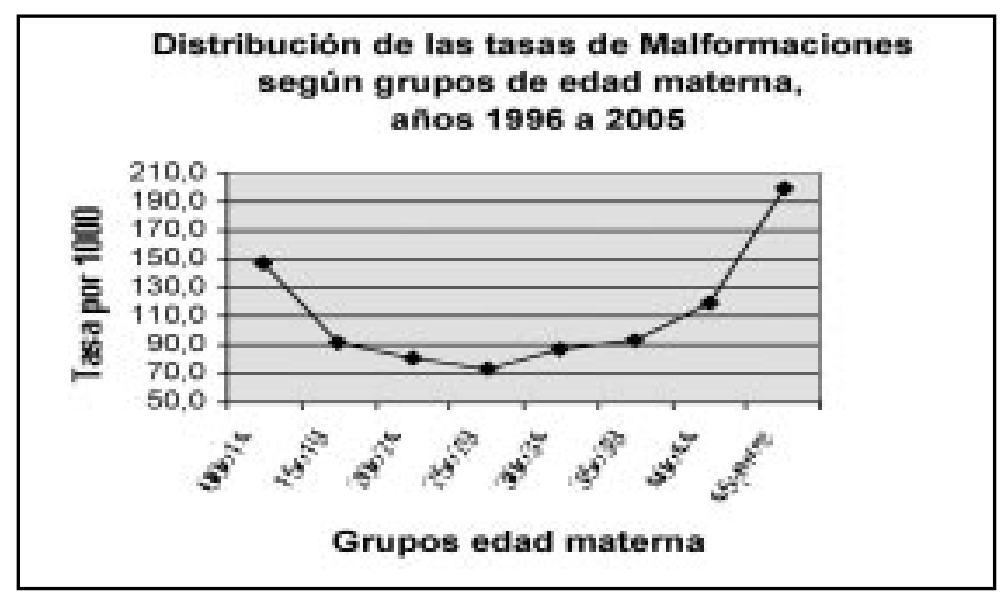

Figura 1. Tasas de prevalencia al nacimiento de malformaciones congénitas según edad materna

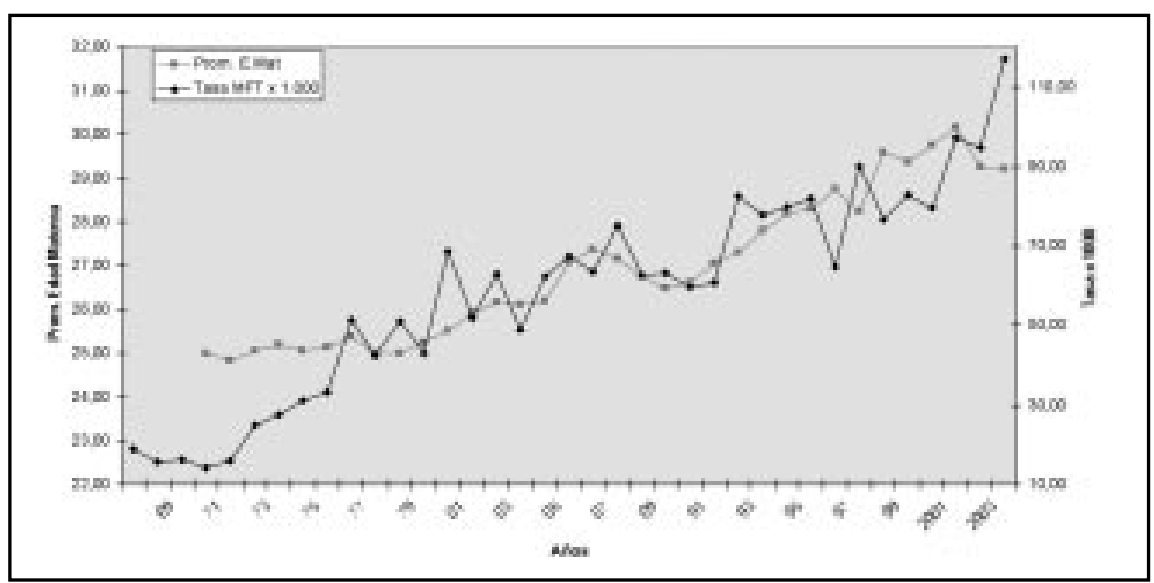

Figura 2. Evolución de los promedios de edad materna y de tasas de prevalencia de malformaciones congénitas. Años 1969 a 2005 


\section{DisCUSIÓN}

Se encontró, al construir y superponer las curvas de evolución en el tiempo de los promedios de edad materna y de tasas de prevalencia al nacimiento de malformaciones congénitas en la maternidad del HCUCH, que a medida que aumenta el promedio de edad materna, se incrementan casi en forma paralela las tasas de prevalencia de MC. En efecto, en 1972 el promedio de edad materna era de 25 años y la tasa de MC en 1970 era 15,8 mil nacimientos. En la actualidad el promedio de edad materna es de 29,2 años y la tasa de MC de 117 por mil nacimientos. En otras palabras el promedio de edad materna se incrementó en 8,6\% en los últimos 25 años, mientras que la tasa de MC aumentó 7,4 veces $(740 \%)$. Esto significa que pequeñas variaciones en los promedios de edad materna provocan grandes cambios en las tasas de MC.

Este hecho ha sido documentado por nosotros en publicaciones anteriores ${ }^{23-26}$

Nuestros resultados en más de 20.000 nacimientos en la maternidad del HCUCH, parecen demostrar que las mujeres de los extremos, dentro de la distribución por edades, tienen un riesgo aumentado de tener hijos portadores de MC, en relación con las mujeres de edades intermedias. Si observamos la Figura 2 se puede apreciar que la distribución de las tasas de prevalencia al nacimiento de MC forman una curva en "U", donde las tasas más bajas está en el grupo de 25 a 29 años y las más altas, entre las madres mayores de 39 años y menores de 20 años. Croen y Shaw ${ }^{8}$ presentan una distribución muy similar a la nuestra en una muestra de más de un millón de nacimientos en California, EEUU. No se demostró diferencia significativa en la incidencia de malformaciones mayores ni de menores entre los grupos de mujeres adoles-

\section{REFERENCIAS}

1. ECLAMC: 34 años de vigilancia epidemiológica del Síndrome de Down en el Hospital Clínico de la Universidad de Chile. 1972-2005. Rev Méd Chile 2006; 134: 1436-44.

2. Congenital Malformation Worldwide. A annual report from the International Clearinghouse for Birth Defects Monitoring Systems. Elsevier Scien- centes y de las mayores de 40 años. Sin embargo, al tratar de identificar qué malformaciones se presentan con mayor frecuencia en los grupos de edades maternas extremas, no encontramos pacientes con síndrome de Down entre los RN de madres menores de 20 años mientras que encontramos un RN con síndrome de Down por cada 33 nacimientos del grupo de mujeres mayores de 39 años. Es importante destacar que a medida que aumenta el promedio de edad materna, también aumenta el riesgo de tener un hijo con síndrome de Down y de otras trisomías, como 13 y 18 . El riesgo de tener un hijo con trisomía 21 va aumentado de 1 en 600 nacimientos en el grupo de 20 a 29 años a $1 / 164$ nacimientos en el grupo de 35 a 39 años y $1 / 33,1$ nacimientos en el grupo de mujeres mayores de 39 años. En una comunicación reciente ${ }^{1}$ demostramos que la recta de regresión mínimo cuadrática de la tasa de prevalencia al nacimiento de síndrome de Down sobre los promedios de edad materna de la población general atendida en la maternidad del Hospital Clínico de la Universidad de Chile, tiene una pendiente que incrementa en 0,475 por 1.000 la tasa de síndrome de Down por cada año que aumenta el promedio de edad materna.

Se encontró que las malformaciones del sistema gastrointestinal ( $p=0,02)$, malformados múltiples y síndrome de Down ( $\mathrm{p}=0,03)$ fueron más frecuentes entre las mujeres de edad mayor y que las malformaciones craneofaciales y gastrosquisis fueron más frecuentes entres las madres adolescentes ( $p=0,02$; OR: 1,5; IC al 95\%: 1,04-2,17) .

El promedio de edad materna de los $\mathrm{RN}$ portadores de aberraciones cromosómicas fue de 34,6 años, mientras que la de los niños controles fue 26,6 años. El promedio de edad materna en los pacientes con gastrosquisis en nuestra muestra fue de 24,2 años.

ce Publishers Co. Inc. New York 1993.

3. Silva C, Nazer J, Fernández P. Defectos de la pared abdominal. Rev Pediatría (Santiago) 1996; 39: 74-7.

4. Pennan DG, Fisher RM, Noblett HR. Increase in incidence of gastroschisis in the South West of England in 1995. Br J Obstet Gynaecol 1998; 105: 228-31.

5. Pardo RA, Nazer J, Cifuentes L. Incidencia de defectos congénitos y bajo peso de nacimiento 
en madres adolescentes. Rev Méd Chile 2003; 131: 1165-72.

6. Centers for Desease Control. Congenital surveillance U.S. Department of Health and Human Service. Teratology 1993; 48: 545-82.

7. Reefhuis J, Honein M. Maternal age and non chromosomal birth defects. Atlanta 1968-2000: Teenager or thirty-something. Who is the risk? Birth Defects Research 2004; (Part A) 70: 572-9.

8. Croen LA, Shaw GM. Young maternal age and congenital malformations: a Population-Based Study. Am J Public Health 1995; 85: 710-13.

9. Abma JC, Chandra A, Mosher WD, Peterson ls, Piccinino LJ. "Fertility, Family Planning, and Women's Health: New Data From the 1995 National Survey of Family Growth". Vital \& Health Statistics-Series 23, Data From the National Survey of Family Growth 1997; (19): 1-114.

10. Sherman SL, Freeman SB, Aluen EG, Lamb NE. Risk factors for nondisjunction of trisomy 21. Cytogenetic Genome Res 2005. 111: 273-80.

11. Kazaura Method R. Lie Rolv T, Irgens Lorentz M, Didriksen Allan, Kapstad Mariann, Egenas John, BJERKEDAL TOR. Increasing Risk of Gastroschisis in Norway: An Age-Period-Cohort Analysis. Am J Epidemiol 2004. Vol. 159, № 4.

12. Hollier LM, Leveno KJ, Kelly MA, MCIntire DD, Cunningham FG. Maternal Age and Malformations in Singleton Births. Obstet Gynecol 2000, 96 (5 Pt 1): 701-6.

13. Lubinsky Mark S. Association of prenatal vascular disruptions with decreased maternal age. Am J Med Genet 1997; 69: 237-9.

14. Lamb Neil E, Yu Kai, Shaffer John, Feingold Eleanor, Sherman Stephanie L. Association between Maternal Age and Meiotic Recombination for Trisomy 21. Am Hum Genet 2005; 76: 91-9.

15. Kovaleva Natalia V, Mutton David E. Epidemiology of Double Aneuploidies Involving Chromosome
21 and the Sex Chromosomes. Am J Med Genet 2005, 134A: 24-32.

16. Vieira Alexandre R, Castillo Taucher Silvia. Edad materna y defectos del tubo neural: evidencia para un efecto mayor en espina bífida que anencefalia. Rev Méd Chile 2005; 133: 62-70.

17. Siega-Riz A, Olshan A, Werler M, Moore C. Fat Intake and the Risk of Gastroschisis. Birth Defects Research 2006. Part A 76: 241-5.

18. Stevens CA, Dobyns WB. Septo-optic dysplasia and amniotic bands: further evidence for a vascular patogenesis. Am J Med Genet A 2004; 15 : 125(1): 12-6.

19. Baird PA, Sudovnik AD, Yee IM. Maternal age and birth defects: a population study. Lancet 1991; 337: 527-30.

20. Fraser Alison M, Brockert John E. Ward R.H. Association of young maternal age with adverse reproductive outcomes. N Engl J Med 1995; 332: 1113-7.

21. Ministerio de Salud. Informe de Estadísticas vitales. 2003. http:// www.minsal.cl/

22. ECLAMC: Manual Operacional RAE 36 ECLAMC. Ed Fiocruz. 2004. Río de Janeiro. Brasil.

23. Nazer J, Eaglin MA, Cifuentes L. Incidencia del Síndrome de Down en la maternidad del Hospital Clínico de la Universidad de Chile. Un Registro de 25 años. Rev Méd Chile. 1998; 126: 383-90.

24. Nazer J, Cifuentes L, Ruiz G. ¿Están aumentando las malformaciones congénitas? Estudio comparativo de los períodos 1971-1977 y 1982-1991 en la maternidad del Hospital Clínico de la Universidad de Chile". Rev Méd Chile 1993; 121: 1068-74.

25. Nazer J, Hubner Me, Cifuentes L. Aumento de la incidencia del Síndrome de Down y su posible relación con el incremento de la edad materna. Rev Méd Chile. 1991; 119: 465.71.

26. Nazer J, Cifuentes L, Ruiz G. Edad materna como factor de riesgo para malformaciones congénitas. Rev Méd Chile 1994; 122: 299-303. 